\title{
Entrevista con el Profesor Stephen J. Ball
}

\author{
Prof. Dr. Jefferson Mainardes ${ }^{1}$ \\ Universidade Estadual de Ponta Grossa \\ Traducción: Prof. Dr. Jorge Gorostiaga ${ }^{2}$ \\ Universidad San Martin \\ Argentina
}

Stephen J. Ball es uno de los más renombrados investigadores del campo de la política educativa en la actualidad. Hasta julio de 2015, ocupó la cátedra de Karl Mannheim Professor of Sociology of Education en la UCL - Institute of Education (Londres). A partir de septiembre de 2015, fue distinguido con el Distinguished Service Professor of Sociology of Education. Es miembro de la British Academy (Academia Nacional de Humanidades y Ciencias Sociales). Stephen J. Ball posee una vasta lista de artículos, libros, capítulos, entrevistas ${ }^{3}$. Esta entrevista fue realizada en Londres, el día 21 de septiembre de 2015. Las preguntas fueron divididas en tres secciones: cuestiones epistemológicas de la política educativa, formación de futuros investigadores y elaboración de artículos de política educativa.

JM: En 2010, fue creada la Red Latinoamericana de Estudios Epistemológicos en Política Educativa (ReLePe) ${ }^{4}$. El principal objetivo de esa red es promover los estudios teóricos y epistemológicos en política educativa. ¿Cuál es su opinión sobre el desarrollo de los estudios teóricos de política educativa? ¿Hay aún lagunas relevantes?

SJB: Pienso que la teoría es muy importante para el estudio de las políticas públicas. Necesitamos más y mejor teoría. Pienso que la mayoría de los análisis de políticas, ahora e históricamente, no han sido muy sofisticados teóricamente o, de hecho, en muchos casos, no se basan en ninguna teoría. Eso significa que muchos de los análisis de políticas incorporan a sus trabajos presupuestos implícitos sobre cómo funciona el mundo, sobre qué es la política, sobre el trabajo de los formuladores de políticas, sobre procesos políticos, esas cosas se dan por sentadas, no son discutidas - humanismo no reflexivo, positivismo simple, ingenuidad sobre el lenguaje - y eso significa que la forma en que las personas piensan e investigan sobre la investigación en políticas se construye

\footnotetext{
${ }^{1}$ Entrevista y notas explicativas: Prof. Dr. Jefferson Mainardes - Universidade Estadual de Ponta Grossa. E-mail: <jefferson.m@, uol.com.br>.

${ }^{2}$ Traducción: Prof. Dr. Jorge Gorostiaga. UNSAM-CONICET. E-Mail: <jorgegoros@gmail.com>

${ }^{3}$ En el sitio del Grupo de Pesquisa Políticas Educacionais e Práticas Educativas (www.uepg.br/gppepe), hay una lista de textos y entrevistas de Stephen J. Ball publicada en portugués (2 libros, 10 capítulos, 9 artículos y 3 entrevistas) y también hay una lista de referencias de investigaciones y publicaciones brasileras que emplean ideas de Ball. 
en forma distorsionada. Por ejemplo, la mayoría de los análisis de políticas es dominado por un presupuesto implícito no discutido de racionalidad, de que los procesos de políticas son racionales, de que es un proceso ordenado, coherente. Y pienso que eso trae distorsiones al trabajo empírico de muchos investigadores, ellos asumen esa racionalidad cuando se involucran con la política. Y mucho del trabajo en política pública no es muy racional, no es muy ordenado, no está muy bien organizado. Por eso tenemos que pensar teóricamente sobre la posibilidad de la irracionalidad, de la confusión, del desorden, del caos. Eso también vale para cuestiones sobre qué es lo que cuenta como dato y sobre qué puede ser considerado un dato. Esto significa que necesitamos pensar en la base ontológica de la política pública, necesitamos pensar sobre la relación de la política pública con el modo en como pensamos que funciona el mundo social de forma general. Así, la teoría es muy importante. Por otro lado, hay ahora un conjunto muy interesante de trabajos, particularmente en Australia, que está re-trabajando la base ontológica de la investigación en política pública, llevado a cabo por personas como Matthew Clarke, Taylor Webb, Kalervo Gulson y Greg Thompson 5 .

JM: En su artículo "Policy Sociology and Critical Social Research: a personal review of recent education policy and policy research" $(1997)^{6}$, menciona dos tipos de epistemologías: epistemología profunda y epistemología de superficie. ¿Podría explicar esos conceptos? En su opinión, ¿es posible explorar la epistemología profunda en un conjunto de publicaciones de política educativa o incluso centrarse solo en un autor?

$\mathrm{SJB}$ : Lo que quiero decir con la epistemología profunda tiene que ver con lo que dije en la anterior respuesta, que la política pública es un proceso social, un proceso relacional, un proceso temporal, discursivo. Es un proceso investido de relaciones de poder, es un proceso político. Una epistemología profunda se involucraría con esos tipos de problemas y cuestiones como base para el proceso de interpretación de la política pública. Entonces, ¿sobre qué presupuestos de poder, de subjetividad, de verdad, el analista de política opera? Esas cosas entran en juego en relación con cualquier estudio, de una forma o de otra. Nuevamente, como dije antes, muchas veces, esas cosas son implícitamente construidas en estudios de políticas públicas, y nunca son abordadas directamente. Reivindicaciones no explícitas de humanismo, logocentrismo,

\footnotetext{
Una de las obras que podría ser citada es el libro Education Policy and Contemporary Theory: implications for research (GULSON; CLARKE; PETERSEN, 2015). Es interesante destacar que, en la introducción del libro, los autores indican que fue escrito por investigadores y estudiantes interesados en el potencial crítico y creativo de la teoría social en el análisis de las políticas. Los objetivos del libro son: argumentar acerca de la utilidad y necesidad de la teoría; celebrar el placer y recompensas de la teoría y ofrecer modelos de uso de la teoría en la investigación sobre políticas educativas. Indican también que el libro complementa la literatura existente sobre el valor de la teoría en la investigación educativa (ANYON, 2009; BALL, 1995; DIMITRIADIS; KAMBERELIS, 2006: DRESSMAN, 2008; SIKES, 2006). Del mismo modo, la preocupación central de la ReLePe es la profundización de los estudios teóricos y epistemológicos en política educativa

${ }^{6}$ Este artículo fue publicado en lengua portuguesa en la Revista Currículo sem Fronteiras, en 2006 (v. 6, n.2, p. 10-32) con el título Sociologia das políticas educacionais e pesquisa crítico-social: uma revisão pessoal das políticas educacionais e da pesquisa em política educacional. Fue también incluido en el libro Políticas Educacionais: questões e debates (BALL; MAINARDES, 2011). Destacando la publicación del libro Educação Global S.A (BALL, 2014).
} 
patriarcalismo y representación son introducidas de contrabando. Y así terminamos con una especie de epistemología de superficie, un conjunto de reflexiones relativamente mundanas sobre el acceso a datos, o el status de la interpretación del actor, la validación del entrevistado, etc. Eso es bueno, pero no avanza lo suficiente. Uno tiene que pensar más allá de eso o pensar más profundamente que eso. ¿Con qué tipo de sujetos suponemos que estamos lidiando aquí? ¿Cuál es la base discursiva de su interpretación? ¿Tenemos una visión del mundo que es una visión constructivista -la idea de que el mundo emerge de los significados de los individuos- o es una visión estructural - existen algunas bases discursivas o estructuras económicas profundas para el mundo que estamos estudiando? Y esto, de nuevo, plantea preguntas sobre qué es lo que cuenta como dato, y cómo vamos a "escribir" los datos, cómo representamos sentido. Es en ese nivel que pienso que uno se involucra con una epistemología profunda. Entonces, uno puede ver casi cualquier trabajo y puede pensarlo en esos términos y observar tanto presupuestos implícitos como explícitos sobre cómo son las personas, cómo funciona el mundo o qué significan las políticas.

JM: La cuestión es que, cuando intentamos analizar artículos y publicaciones de políticas educativas en términos epistemológicos, en algunas ocasiones nos enfrentamos a dificultades y desafíos porque, de modo general, los autores no explicitan aspectos epistemológicos de su investigación. Hemos intentado desarrollar metainvestigación, pero ha sido una tarea compleja y difícil'. En su artículo "What is policy? 21 years later: reflections on the possibilities of policy research" $(2015)^{8}$ usted escribe: "Tendemos a limitar nuestra ambición y quedarnos en la superficie de las cosas, tomando el valor aparente de una política y re-inscribiendo sus peticiones de coherencia en nuestros análisis, en lugar de buscar abordar El Orden de las Cosas [Las Palabras y las Cosas] (Foucault, 1970)". Esa reflexión ¿puede ser tomada para el campo de la política educativa en general?

SJB: Sí, pienso que sí. La frase "el orden de las cosas" es una alusión al libro de Foucault Las Palabras y las Cosas. Y lo que yo estoy sugiriendo es que tal vez necesitamos pensar epistémicamente en el sentido de Foucault. Tenemos que pensar sobre las epistemes que sustentan y tornan posibles ciertas afirmaciones sobre la verdad de la política pública. Los fundamentos sobre los que las declaraciones son consideradas verdaderas o falsas. Y, en ese momento, se puede argumentar... he pensando un poco sobre eso... tomando como base el trabajo de Foucault, que estamos entrando ahora en una especie de cuarta episteme. El habló sobre tres períodos epistémicos en Las

\footnotetext{
${ }^{7}$ Más información sobre el enfoque de las epistemologías de la política educativa y metainvestigación puede encontrarse en Tello (2012) y Tello y Mainardes (2012, 2015)

${ }^{8}$ El título del artículo es "Qué es la política pública? Veintiún años después: reflexiones sobre las posibilidades de la investigación sobre políticas públicas. En inglés, fue publicado en Discourse: studies in the cultural politics of educations, v. 36, n. 3, p. 306-313, 2015 .

${ }^{9}$ Publicado en inglés como "The order of things". Nota del traductor.
} 
Palabras y las Cosas, culminando en la modernidad. Sin embargo, tal vez, al pensar sobre algunos de sus trabajos posteriores, estamos ahora dentro de una episteme neoliberal. Y, dentro de eso, hay ciertas premisas sobre la verdad, y que en forma esencial y simple las afirmaciones que son tomadas como verdaderas son aquellas que se refieren al funcionamiento del mercado -tienen que ver con la competencia y la elección, la inversión y la responsabilidad, la productividad y la eficiencia. Esto implica mirar más allá de las características superficiales de la política pública, para comenzar a entender esas características epistémicas neoliberales de las políticas. Así que es eso lo que estoy sugiriendo.

JM: En la ReLePe estamos también interesados en analizar la constitución del campo de la política educativa en nuestros países (Brasil, Argentina, Colombia, Chile, México, etc.). ¿Usted considera que la política educativa puede ser considerada un campo de conocimiento específico? Algunos investigadores la consideran como una disciplina o como parte de la sociología de la educación (como parece ser el caso en el Reino Unido) ${ }^{10}$.

SJIB: Es una cuestión muy difícil, porque la sociología de la educación es ahora un campo muy dispar de conocimiento, no hay mucha coherencia, es una combinación muy laxa de toda una gama de diferentes tipos de trabajos, diferente puntos de foco, niveles de análisis, perspectivas teóricas, ontologías. No creo que haya nada que se pueda capturar o describir en una etiqueta tan simple o en un descriptor como "sociología de la educación". Y, si se mira en la otra dirección, sí, se puede hablar sobre el estudio de las políticas educativas como un campo de investigación, pero inmediatamente plantea la pregunta sobre la relación de la política educativa con otros tipos de políticas y otras posibilidades analíticas --por ejemplo, los analistas de políticas educativas no son buenos lidiando con la cuestión del dinero- y podríamos querer mirar a Harvey, Larner, Peck y Brenner y Jessop, para elaborar ideas sobre la "economización" y la geografía de la política. Particularmente, si se toma muy en serio el neoliberalismo, como Tony Blair señalaba cuando él era primer ministro en el Reino Unido, "la educación es nuestra mejor política económica", en ese sentido, se puede argumentar que el análisis de políticas educativas es, en verdad, una especie de subconjunto de análisis de la política económica. Entonces, se podría argumentar en forma más coherente en cuanto a la relación de la política educativa con otros tipos de análisis político, más que con la sociología de la educación. Pero eso también depende de tradiciones nacionales y de la organización del conocimiento, y sus relaciones verticales y horizontales, como Basil

\footnotetext{
${ }^{10}$ Hasta el presente (2015), no hubo en Brasil y en América Latina un debate en torno de la cuestión si la política educativa es un campo específico del conocimiento o un campo académico. En la literatura internacional, autores como Cibulka (1994), Ladwig (1994), Lingard, Rawolle y Taylor (2005) han considerado a la política educativa como un campo. Más recientemente, Lingard y colaboradores, con base en la teoría de los campos sociales de Bourdieu, consideran que hay un campo global de las políticas educativas (ej.: RAWOLLE; LINGARD, 2015; SELLAR; LINGARD, 2014), o bien como políticas educativas globales. Azevedo e Aguiar (2001), Mainardes (2009); Santos e Azevedo (2009, 2012), Krawczyk (2012), Tello (2012, 2013, 2014), Schneider (2014), Souza (2014) también se refieren a la política educativa como campo. Tello (2013) se refiere a la política educativa como campo teórico. Oliveira (2011) se refiere a las políticas públicas en educación.
} 
Bernstein señalaría, se organizan en determinado lugares. Entonces, es difícil responder a eso. Igualmente, hay que pensar si esta es una pregunta importante. ¿Es importante que existan distinciones claras y demarcaciones entre distintas áreas de investigación? O, quizás, puede ser más productivo minimizar esas distinciones y pensar de una forma pos-disciplinar, pos-filosófica sobre la teoría y el análisis, aprovechando y reuniendo conocimientos de diferentes teorías y disciplinas, conjugándolas en una teorización más plural y diversificada.

JM: Una de las cuestiones que ha surgido de las discusiones con investigaciones de la ReLePe está vinculada al objeto de estudio de la política educativa. En su opinión, ¿cuál es el objeto (o los objetos) de estudio de la política educativa?

SJIB: El objeto de estudio es construido por la epistemología con la cual uno opera. Por un lado, puede ser que uno esté interesado en los significados y en las interpretaciones de los actores de la política. O uno podría estar interesado en los principios epistémicos que dan origen a la verdad y a la falsedad de las políticas y otras cosas en el medio. Es una pregunta sobre el tipo de decisiones que uno toma en cuanto a su posición epistemológica. Eso, entonces, construye el objeto de estudio, de la forma en que Foucault sugiere que los cuerpos de conocimiento construyen los objetos sobre los cuales hablan. Un claro ejemplo en la investigación de política es la "implementación". Hay una tradición firmemente arraigada de investigación sobre implementación que interpreta la política como un proceso hiper-racional y lineal al "final" del cual encontramos "fallas" de implementación. Esa es una concepción de la política a la que yo intento oponerme y ridiculizar en How Schools do Policy ${ }^{11}$, frente a la cual intento construir el objeto de estudio de un modo totalmente diferente, como enactment ${ }^{12}$.

JM: Tal vez sea difícil definir un fijar un objeto?

SJIB: Diferentes versiones de estudios de políticas construyen el objeto de conocimiento de manera diferente en formas muy variadas. En el Reino Unido, el análisis de políticas, por muchos años, se centró en el trabajo del gobierno, y la política pública fue vista casi como sinónimo de gobierno, en el sentido tradicional de la palabra. En tanto, ahora, comprendemos la política de formas diferentes, y yo me siento tentado de argumentar que la política es construida y hecha en todos los tipos de arenas, en

\footnotetext{
How do schools do policy: policy enactments in Secondary Schools, de Stephen J. Ball, Meg Maguire, Annette Braun, publicado en 2012 por la Editora Routledge.

${ }^{2}$ El término 'policy enactment' es de difícil traducción. En una entrevista con el Prof. Stephen J. Ball publicada en 2009 , explicamos que "esta palabra ha sido empleada en el contexto legal para describir el proceso de aprobación de leyes y de decretos. En la entrevista, Ball usó la palabra en el sentido teatral, refiriéndose a la noción que el actor posee sobre un texto que puede ser presentado/representado de diferentes formas. El texto, es apenas una pequeña parte (sin embargo, una parte importante) de producción. Ball usó ese término para indicar que las políticas son interpretadas y materializadas de diferentes y variadas formas. Los actores involucrados (por caso, los profesores) tienen el control del proceso y no son 'meros implementadores' de las políticas (MAINARDES; MARCONDES, 2009). Rosa (2012) considera que la mejor traducción de policy enactment es "escenificación de las políticas". Hemos también empleado el concepto de "política en acción” para referirnos a la políticas que está siendo efectivamente desarrollada en la escuela.
} 
todos los tipos de niveles, por todo tipo de personas; de forma que el objeto de estudio se torna difuso o tiene que ser entendido en términos de un análisis de trayectoria; como moviéndose por el tiempo y por el espacio. La manera como construimos nuestro objeto de estudio también anticipa los diseños y los métodos de investigación que usamos para trabajar con él.

JM: Ahora, tengo una pregunta sobre "big and small theories" (grandes y pequeñas teorías), lo que considero una idea importante. ¿Podría usted explorar un poco más su idea de teorías grandes y pequeñas y cómo la ha aplicado en sus investigaciones?

SJIB: Bien, eso trae nuevamente lo que yo quiero decir con epistemología profunda y superficial. Clásicamente, small theory (teoría pequeña) es una teoría interpretativa. Es sobre los actores y sus relaciones. No estoy diciendo que no es importante; creo que es importante entender cómo los actores le dan sentido a la política y cómo, entonces, actúan en relación a ese sentido dado. Ciertamente, este se está tornando un abordaje muy común, y también muy dominante en el análisis de políticas, y también tiene una larga historia en base a la realización de entrevistas con los formuladores de políticas, etc. Sin embargo, por otro lado, big theory (teoría grande) lleva en direcciones ligeramente diferentes, generalmente en algún tipo de dirección estructuralista. Y, obviamente, el marxismo es una teoría grande, que ve la elaboración de políticas como fundamentalmente dependiente del equilibrio de fuerzas dentro de la lucha de clases, $o$ relacionada a determinada constitución de los medios de producción en un determinado período de tiempo. Del mismo modo, la teoría del discurso va en dirección al tipo de cuestiones epistémicas a las cuales me referí anteriormente, lo que incluye una mirada de los fundamentos sobre los cuales los alegatos sobre la verdad son justificados, y a cómo se producen los sujetos de las políticas, cómo son "hablados" por el discurso, cómo son formados y reformados por la política e invitados o convocados a hablar, actuar, leer, trabajar, pensar, sentir, comportarse, valorizar, desear. Esto es, las formas por las cuales la política es construida en lenguaje, por medio de prácticas y relaciones sociales específicas -relaciones de poder-y de formas y estructuras organizacionales específicas. Entonces, es eso lo que quiero decir con teorías más grandes -es mucho más ambicioso, tanto en su profundidad como en su amplitud, pues incluye llevarnos en dirección a lo que Foucault llamaría un dispositif o un apparatus, o lo que Deleuze denomina 
assemblage $^{13}$. Las políticas son assemblages (ensamblajes) inestables de valores, autoridad, significado y práctica, que reúnen varios estados de cosas y organismos, tanto como declaraciones, modos de expresión, y regímenes enteros de signos -tanto objetos materiales cuanto inmateriales. Entonces, uno no puede preocuparse simplemente por una tecnología o una política, sino que uno tiene que ver esas cosas en una relación compleja de objetos, de personas, de prácticas del lenguaje, en tanto se relacionan entre sí como en una especie de un todo más o menos coherente. Esta no es una rearticulación de lo macro y lo micro, sino una eliminación de ese binario para ver la política como un conjunto de técnicas, categorías, objetos y subjetividades. Esta es una concepción muy material del discurso que en vez de priorizar la "lectura" de los textos, lidia tanto con las condiciones de posibilidad y contingencia histórica cuanto con la manera en que el discurso es inscripto en los cuerpos.

JM: Con la ReLePe hemos organizado tres eventos. El último fue el I Encuentro Latinoamericano de Profesores de Política Educativa ${ }^{14}$, que se realizó en la UNIFESP, en Guarulhos, los días 6 y 7 de julio de 2015. Una de las cuestiones discutidas en el evento fue la formación (maestría, doctorado, etc.) de investigadores para el campo de la política educativa. En su opinión, ¿qué aspectos son relevantes para ser considerados en la formación de los futuros investigadores en política educativa?

SJB: Pienso que la respuesta simple para eso es: "hacerlos leer teoría". Lo que observo frecuentemente con mis alumnos investigadores es que ellos vienen interesados en un objeto de estudio, están interesados en la privatización, o en la elección de escuela, o en la introducción de las relaciones de gerenciamiento para la gobernanza de la educación superior, o en la situación de los estudiantes de clase trabajadora en la universidad de elite, tienen un tema de estudio y lo conciben de una forma bastante directa, empírica. Mis estudiantes piensan: "voy a preguntar a algunas personas sobre eso y ellos me dirán cómo es". Sin embargo, muchas veces, cuando yo les hago leer teoría, ellos, en verdad acaban repensando, de algún modo, todo su proyecto, y a

\footnotetext{
De acuerdo con Wise (2005, p. 77), “assemblage (agenciamiento), como es empleado en la obra de Deleuze y Guattari es un concepto que lee el juego de la contingencia y estructura, organización y cambio" (p. 77). El término en francés es agencement, traducido normalmente como "juntar", "acordar", disponer, "disposición" o "encajar". Es importante destacar que agencement "no es un término estático, no es un acuerdo o una organización, más bien es el proceso de acordar, organizar, junta (...) Assemblage no es un conjunto de partes predeterminadas o una estructura ya concebida (un modelo). Tampoco assemblage es una colección aleatoria de cosas, ya que hay un sentido de que assemblage es un todo de algún tipo que expresa alguna identidad y reivindica un territorio. Assemblage es un devenir que reúne elementos" (WISE, 2005, p. 77). Youdell (2015) explica que Deleuze y Guattari emplean nociones de "agrupamientos", "acuerdos" y "assemblages" "para pensar la multiplicidad de elementos diversificados y móviles que se combinan para constituir formaciones sociales complejas. Ellos sugieren que aparentemente entidades enteras, y la 'educación' puede ser tal entidad, pueden ser entendidas como assemblages de componentes heterogéneos que atraviesan el orden económico, político, estatal, social, institucional, lingüístico, semiótico, representacional, discursivo, subjetivo y afectivo. Tales componentes han sido, muchas veces, tratado como separados o con importancia diferencial en ciencia sociales, sin embargo, para Deleuze y Guattari (1983: 52), ellos son inseparables" (YOUDELL, 2015, p 111).

${ }^{14}$ Los anales del I Encuentro Latinoamericano de Profesores de Política Educativa están disponibles en www.encuentrorelepe. com.br. Información sobre las Jornadas Latinoamericanas de Estudios Epistemológicos en Política Educativa, realizadas en 2012 (Buenos Aires) y 2014 (Curitiba) se pueden ver en: www.relepe.org.
} 
veces, re-conceptualizan sus objetos de estudio. Entonces, pienso que realmente lo más importante es ser teóricamente "alfabetizado" y ser capaz de pensar más allá del objeto de estudio, cómo conceptualizarlo. Y eso se puede transponer, porque uno puede entonces usar sus recursos teóricos para otros estudios que abordan diferentes temas y problemas. Eso no significa que uno tenga que comprometerse con una posición teórica. Uno puede desarrollar una "caja de herramientas" con conceptos, técnicas y métodos de teorías diferentes, siempre y cuando tenga algún grado de coherencia en su trabajo. Uno puede emplear esa "caja de herramientas" en relación a cualquier objeto de estudio. Entonces, pienso que esa es la preparación más importante para el análisis de políticas, para los estudiantes de investigación de políticas... que ellos lean mucha teoría. Mi experiencia también es que, si los alumnos leen mucha teoría, normalmente terminan encontrando algo que tiene sentido para ellos, y eso es lo importante. No veo eso como una especie de compromiso, como una identidad. Cuando yo me formé como investigador, uno tenía una identidad que se basaba en un compromiso teórico. Entonces, uno podía ser un neomarxista, o un interaccionista simbólico o una feminista. Creo que esas delimitaciones ya no son tan claras, sino que es más importante tener algún grado de reflexividad sobre las decisiones que uno toma. $\mathrm{Y}$ también adquirir y desarrollar un conjunto de herramientas teóricas que funcionen, que sean útiles, que tengan poder en relación a aquello que uno está tratando de entender.

JM: Tuve la oportunidad de oír sus respuestas a las preguntas que se encuentran en el sitio del Journal of Education Policy ${ }^{15}$ que se emplean para presentar el Journal y para orientar a los colaboradores. En su opinión, ¿cuáles son las principales características de un buen artículo sobre política educativa?

SJIB: Esa es una pregunta difícil, porque un artículo podría ser formulado con diferentes objetivos. Podría ser un intento de desarrollar una perspectiva teórica en relación al análisis de políticas. O podría ser un artículo de trabajo empírico. Pero, si es un artículo empírico, entonces, tomando como base lo que vengo diciendo, debería ser algo que permita al lector comprender la base sobre la que el autor construyó su objeto de estudio y pueda ver las formas por las cuales esos presupuestos, esas premisas, habilitan y limitan al investigador en términos de lo que son capaces de decir sobre sus objetos de estudio. Entonces, para mí, en el otro extremo, una de las cosas importantes es tener un grado de modestia sobre lo que uno es capaz de conseguir y sobre qué afirmaciones uno es capaz de hacer. Pienso que muchos investigadores de políticas públicas exageran el valor de sus investigaciones, hacen afirmaciones que no están muy fundamentadas, ni empíricamente y ni, ciertamente, epistemológica o teóricamente. Entonces, creo que es preciso tener más reflexividad en relación a tales artículos, que es

El Journal of Education Policy fue creado en 1986. Es una de las revistas más importantes en política educativa. En el sitio http://www.tandfonline.com/loi/tedp20, el profesor Stephen J. Ball, en calidad de editor, responde una serie de preguntas sobre la revista. 
lo que Bourdieu, por ejemplo, siempre defendió, que es necesario tener una comprensión de la base sociopolítica de la producción del trabajo intelectual. Y Bourdieu también era muy claro en que lo que él estaba haciendo era un emprendimiento muy modesto -el habló no sobre desarrollar teorías, sino sobre hacer conjuntos de experiencias. Y él nunca alegó haber producido una gran teoría social, del mismo modo que Foucault nunca reivindicó haber escrito una teoría social. Foucault hablaba sobre la construcción de conocimiento estratégico como de a un ladrillo por vez. Es eso lo que pienso que necesitamos, necesitamos más construcción de paredes y hacer menos afirmaciones sobre la construcción de casas y palacios de baja calidad, sin cimientos... necesitamos ser más modestos... y, entonces, podríamos llegar a algún lugar.

JM: Muchas gracias Profesor Ball.

\section{Referencias}

ANYON, J. Theory and educational research: Toward critical social explanation. London: Routledge, 2009.

AZEVEDO, J. M. L. de; AGUIAR, M. A. A produção do conhecimento sobre a política educacional no Brasil: um olhar a partir da ANPED. Educação \& Sociedade, Campinas, v. 22, n. 77, p. 49-70, set./dez. 2001.

BALL, S. J. Educação global S.A.: novas redes políticas e o imaginário neoliberal. Ponta Grossa: Editora UEPG, 2014.

BALL, S. J. Intellectuals or technicians? The urgent role of theory in Educational Studies. British Journal of Educational Studies, v. 43, n. 3, p. 255-271, 1995.

BALL, S. J.; MAINARDES, J. Políticas Educacionais: questões e dilemas. São Paulo: Cortez, 2011.

CIBULKA, J. G. Policy analysis and the study of the politics of education. Journal of Education Policy, v. 9, n. 5, p. 105-125, 1994.

DIMITRIADIS, G.; KAMBERELIS, G. Theory for education. New York: Routledge, 2006.

DRESSMAN, M. Using social theory in educational research: A practical guide. New York and London: Routledge, 2008.

GULSON, K. N.; CLARKE, M.; PETERSEN, E. B. (Eds.). Education policy and contemporary theory: implications for research. Abingdon: Routledge, 2015.

KRAWCZYK, N. A historicidade da pesquisa em política educacional: o caso do Brasil. Jornal de Políticas Educacionais, Curitiba, v. 6, n. 12, p. 3-11, jul./dez. 2012. 
LADWIG, J. G. For whom this reform?: outlining educational policy as a social field. British Journal of Sociology of Education, v. 15, n. 3, p. 341-363, 1994.

LINGARD, B.; RAWOLLE, S.; TAYLOR, S. Globalizing policy sociology in education: working with Bourdieu. Journal of Education Policy, v. 20, n. 6, p. 759-777, 2005.

MAINARDES, J. Análise de políticas educacionais: breves considerações teóricometodológicas. Contrapontos, Itajaí, v. 9, n. 1, p. 4-16, jan./abr. 2009.

MAINARDES, J; MARCONDES, M. I. Entrevista com Stephen J. Ball: um diálogo sobre justiça social, pesquisa e política educacional. Educação \& Sociedade, v. 30, n. 106, p. 303-318, jan./abr. 2009.

OLIVEIRA, D. A. As políticas públicas em educação e a pesquisa acadêmica. In: DUARTE, A.; OLIVEIRA, D. A. (Orgs.). Políticas públicas e educação: regulação e conhecimento. Belo Horizonte: Fino Traço, 2011, p. 71-89 (Educere, 9).

RAWOLLE, S.; LINGARD, B. Bourdieu and doing policy sociology in education. In: GULSON, K. N.; CLARKE, M.; PETERSEN, E. B. (Eds.). Education policy and contemporary theory: implications for research. Abingdon: Routledge, 2015. p. 15-26.

ROSA, S. S. Reformas educacionais e pesquisa: as políticas "em cena" no Brasil e na Inglaterra. E-curriculum, São Paulo, v. 8, n. 2, p. 1-28, 2012.

SANTOS, A. L. F.; AZEVEDO, J. M. L. A Pós-Graduação no Brasil, a pesquisa em educação e os estudos sobre a política educacional: os contornos da constituição de um campo acadêmico. Revista Brasileira de Educação, Rio de Janeiro, v. 14, n. 42, p. 534-550, set./dez. 2009.

SANTOS, A. L. F.; AZEVEDO, J. M. L. de. Regulação e legitimação da pesquisa sobre a política educacional como campo acadêmico: um estudo a partir dos programas de Pós-Graduação do nordeste brasileiro. In: GOMES, A. M.; OLIVEIRA, J. F. de (Orgs.). Reconfiguração do campo da educação superior. Campinas: Mercado de Letras, 2012. p. 71-93. (Série Estudos em Políticas Públicas e Educação).

SCHNEIDER, M. P. Pesquisa em política educacional: desafios na consolidação de um campo. Revista Educação (PUC-Campinas), Campinas, v. 19, n. 1, p. 5-13, jan./ abr. 2014

SELLAR, S.; LINGARD, B. The OECD and the expansion of PISA: new global modes of governance in education. British Educational Research Journal, v. 40, n. 6, p. 917-936, 2014.

SIKES, P. Towards useful and dangerous theories. Discourse: Studies in the Cultural Politics of Education, v. 27, n. 1, p. 43-51, 2006. 
SOUZA, A. R. A pesquisa em políticas educacionais no Brasil: de que estamos tratando? Práxis Educativa, v. 9, n. 2, p. 355-367, jul./dez. 2014

TELLO, C. Las epistemologías de la política educativa: vigilancia y posicionamiento epistemológico del investigador en política educativa. Práxis Educativa, Ponta Grossa, v. 7, n. 1, p. 53-68, jan./jun. 2012.

TELLO, C. El campo teórico de la política educacional: modelos, abordajes y objetos de estudio. Jornal de Políticas Educacionais, Curitiba, n. 14, p. 62-75, jul./dez. 2013.

TELLO, C. The theoretical field of education policy: characteristics, objects of study, and mediations. A Latin American perspective. American Journal of Educational Research, v. 2, n. 4, p. 197-203, 2014.

TELLO, C. ; MAINARDES, J. La posición epistemológica de los investigadores en Política Educativa: debates teóricos en torno a las perspectivas neo-marxista, pluralista y pos-estructuralista. Archivos Analíticos de Políticas Educativas, v. 20, n. 9, p. 1-31, 2012.

TELLO, C.; MAINARDES, J. Revisitando o enfoque das epistemologias da política educacional. Práxis Educativa, Ponta Grossa, v. 10, n. 1, p. 153-178, jan./jun. 2015.

WISE, J. M. Assemblage. In: STIVALE, C. J. Gilles Deleuze: key concepts. Montreal: McGuill-Queens University Press, 2005. p. 77-87.

YOUDELL, D. Assemblage theory and education policy sociology. In: GULSON, K. N.; CLARKE, M.; PETERSEN, E. B. (Eds.). Education policy and contemporary theory: implications for research. Abingdon: Routledge, 2015. p. $110-212$.

Recebido em: 20/10/2015

Aprovado em: 23/11/2015 